\title{
Serum pNF-H, a potential predictive biomarker for postoperative cognitive dysfunction in elderly subjects undergoing hip joint replacement
}

Huafeng Zhang

Ningbo No 2 Hospital

Jinwei Zheng

Ningbo No 2 Hospital

Ruichun Wang

Ningbo No 2 Hospital

Guorong Wu

Ningbo No 2 Hospital

Junping Chen ( $\nabla$ yunfeiyangtg@126.com )

Ningbo No 2 Hospital https://orcid.org/0000-0003-1381-9966

\section{Research Article}

Keywords: Postoperative cognitive dysfunction, elderly, hip joint replacement, pNF-H, biomarker

Posted Date: January 3rd, 2019

DOl: https://doi.org/10.21203/rs.2.165/v1

License: (9) This work is licensed under a Creative Commons Attribution 4.0 International License. Read Full License 


\section{Abstract}

Background: Postoperative cognitive dysfunction (POCD), which refers to a cognitive impairment subsequent to surgical procedures, is a common complication in the elderly subjects. This study aimed to investigate potential risk factors for POCD in elderly subjects undergoing hip joint replacement.

Methods: Consecutive elderly osteoarthritis patients who were scheduled to undergo hip replacement under epidural anesthesia were enrolled into this single-center, prospective observational study. Serum phosphorylated neurofilament heavy subunit-H (pNF-H) was measured by the enzyme-linked immuno sorbent assay (ELISA) method. A level of $>70.5 \mathrm{pg} / \mathrm{mL}$ was accepted as pNF-H positivity.

Neuropsychological assessment at baseline (one day before the surgery) and postoperative day 7 was conducted. POCD was defined according to the calculated Z scores. Risk factors for POCD were evaluated by univariate and multivariate logistic regression analyses.

Results: In final, 287 patients were enrolled and 55 had suffered POCD within postoperative 7 days with an incidence of $19.2 \%$. The final multiple logistic regression analysis revealed a higher pNF-H positivity was the only independent risk factor for POCD (OR: 2.03, 95\% Cl: 1.21-3.29, P=0.012)

Conclusions: Our results revealed an increased preoperative serum pNF-H expression was an independent risk factor for POCD development in elderly subjects undergoing hip joint replacement, suggesting the close association between anatomical damage in central nervous system (CNS) and POCD.

Key words: Postoperative cognitive dysfunction; elderly; hip joint replacement; pNF-H; biomarker

\section{Introduction}

Postoperative cognitive dysfunction (POCD), a common complication in the elderly subjects, refers to a cognitive impairment subsequent to surgical procedures, especially the decline in executive functions and memory (1). As reported by previous evidence, the incidence of POCD ranges from $8.9 \%$ to $46.1 \%$ with elusive pathophysiology (2). During those patients aged over 60 years undergoing non-cardiac surgery, the incidence of POCD was reported to be $25.8 \%$ at 1 week and $9.9 \%$ at 3 months by the International Study of Postoperative Cognitive Dysfunction (ISPOCD) (3). Due to the elusive pathophysiology and complicated neuropsychological testing requirements, the diagnosis of POCD is always delayed. The onset of POCD is an important cause of prolonged hospital stay, poorer social outcomes, morbidity and mortality after major operations (4). Thus, it is of crucial importance to identify noninvasive and reliable biomarkers for POCD prediction and risk stratification.

Phosphorylated neurofilament heavy subunit-H (pNF-H), a major structural protein in central nervous system (CNS) axons, shows an adequate sensitivity for the spinal cord injury (5). Increased pNF-H expressions have been reported to be significantly associated with CNS damage (6). Among animals, low pNF-H expression is reported closely associated with increased cell death (7). PNF-H is also involved in axonal regeneration and it is suggested as a good measure of brain damage, as well as axonal recovery 
(8). A recent study has suggested the potential application of pNF-H as a biomarker of neural damage among patients undergoing chemotherapy for breast cancer (9). pNF-H is also an effective predictive biomarker for postoperative delirium among patients who underwent surgery for abdominal cancer (10). However, whether it can serve as a predictor for POCD in elderly patients undergoing hip joint replacement remains unclear. This study aimed to identify potential predictors for POCD in elderly patients with hip joint replacement.

\section{Methods}

This study protocol was approved by the Medical Institutional Ethics Committee of Ningbo No. 2 hospital and Zhejiang province. This investigation was a single-center, prospective observational study which was conducted in the Department of anesthesiology from July 2015 to July 2017. Consecutive elderly osteoarthritis patients who were scheduled to undergo hip replacement under epidural anesthesia were enrolled. All the enrolled patients provided written informed consent before the surgery. Inclusion criteria: (1) aged between 65 and 80 years; (2) American Society of Anesthesiologists (ASA) grade II or III; (3) undergoing first-time unilateral total hip joint replacement under epidural anesthesia. The exclusion criteria: (1) with preexisting neurological disease or psychiatric disorder; (2) preoperative Mini-Mental State Examination (MMSE) score <24; (3) with a history of cardiovascular or neurosurgical surgery; (4) with alcohol or drug dependence; (5) inability to read or speak, with vision or hearing impairment; (5) with low compliance; (6) loss to follow-up or with no completed data.

\section{Neuropsychological assessment and POCD definition}

Neuropsychological assessment at baseline (one day before the surgery) and postoperative day 7 was conducted following the guidance of previous consensus statement (11). As widely described by previous reports, the test battery included Digit span test, MMSE, Trail making test (part A), Word recognition memory tests, Verbal fluency test and Symbol digit test (12). According to the guidance of International Study of Postoperative Cognitive Dysfunction (ISPOCD1 and ISPOCD2), a Z score for each test was calculated $(3,13)$. In this study, patients were defined as suffering POCD while at least two Z scores $\geq$ 1.96 (14).

\section{Clinical data collection}

The following perioperative clinical data were recorded: (1) demographic data including age, gender, Body Mass Index (BMI), ASA physical status, smoking habits; (2) Preoperative comorbidities including diabetes, hypercholesterolemia, hypertension, peripheral vascular disease, history of myocardial infarct; (3) preoperative medications including angiotensin-converting enzyme (ACE) inhibitors, $\beta$-blockers, and statins; (4) other clinical data including preoperative MMSE, duration of surgery and anesthesia, recovery time, estimated blood loss, blood transfusion.

\section{Laboratory tests}


Fasting blood samples from all the enrolled patients were obtained at one day before the surgery (baseline). Serum samples were obtained after centrifugation $\left(3000 \mathrm{rpm}, 10 \mathrm{~min}, 4^{\circ} \mathrm{C}\right)$ and then stored at $-80^{\circ} \mathrm{C}$ for further laboratory tests. The inflammatory biomarkers including C-reactive protein (CRP), interleukin-6 (IL-6), tumor necrosis factor-a (TNF-a) were measured by the enzyme-linked immuno sorbent assay (ELISA) method using kits (R\&D Systems, Minneapolis, MN, USA). The serum expression of pNF-H was detected using human pNF-H ELISA kit (BioVendor, Modrice, Czech Republic) following the guidance of the manufacturer's protocol. The obtained serum samples were diluted three fold before the $\mathrm{pNF}-\mathrm{H}$ detection. A level of $>70.5 \mathrm{pg} / \mathrm{mL}$ was accepted as pNF-H positivity according to previous reports (5). The preoperative blood analyses (including hemoglobin, white blood cell) and blood biochemical examinations (including albumin, creatinine, urea) were also detected by the laboratory in our hospital.

\section{Statistical analysis}

GraphPad Prism 5.0 (GraphPad Inc., CA, USA) and SPSS 19.0 (SPSS, Inc., IA, USA) were utilized for the data analysis. Continuous and categorical data were presented as mean and standard error (S.E.M), number ( $\mathrm{n}$ ) and percentage (\%), respectively. Mann-Whitney $\mathrm{U}$ test, $\mathrm{t}$ test, Chi-square test and Fisher exact test were used for data analyses as appropriate. Risk factors for POCD were evaluated by univariate and multivariate logistic regression analyses. All statistical analyses were bilateral probability and a $P$ value of $<0.05$ was considered statistically different.

\section{Results}

\section{Patient characteristics}

According to the inclusion criteria, a total of 351 eligible elderly osteoarthritis patients were screened for the study. 64 were excluded due to the exclusion criteria, 5 with preexisting neurological disease, 7 with a history of cardiovascular surgery, 6 with alcohol dependence, 6 inability to read or speak, 15 with low compliance and 25 with no completed data. In final, 287 patients were enrolled and 55 had suffered POCD within postoperative 7 days with an incidence of $19.2 \%$. Table 1 compares the characteristic of patients with POCD or without POCD development. As shown, no significant differences among gender distribution, BMI, ASA phrase status, smoking habits, preoperative medications, recovery time and blood transfusion were observed between them $(P>0.05)$. Patients with POCD showed a significantly higher age comparing with those without POCD $(71.3 \pm 3.3$ vs $70.2 \pm 2.9, P=0.014)$. The incidences of preoperative comorbidities (diabetes and hypertension) were also statistically different between the two groups $(P=0.028$ and $P=0.046$, respectively). A lower preoperative MMSE score seemed to be associated with an increased risk of POCD development $(P=0.033)$. In respect to the operation indexes, a longer duration of surgery and anesthesia, higher estimated blood loss were observed in patients with POCD $(P<0.05)$.

\section{Laboratory tests}

Table 2 summarizes the results of laboratory tests between two groups. Patients who suffered POCD showed an obviously increased serum expressions of CRP and TNF-a $(P<0.05)$. At the onset of POCD, 10 
$(10 / 55,18.2 \%)$ patients demonstrated pNF-H positivity, which was quite higher than those patients without POCD $(16 / 232,6.9 \%, P=0.009)$.

\section{Risk factors for POCD}

As shown in Table 3, ten potential risk factors mentioned above were included into the univariate and multivariate logistic regression analyses. As illustrated by the univariate analysis, age, preoperative MMSE, CRP and pNF-H positivity were 4 risk factors for POCD $(P<0.05)$. The final multiple logistic regression analysis revealed a higher pNF-H positivity was the only independent risk factor for POCD (OR: $2.03,95 \% \mathrm{Cl}: 1.21-3.29, \mathrm{P}=0.012$ )

\section{Discussion}

POCD is a great attack and challenge for those patients who undergo major surgery, especially for the elderly subjects (15). A review by Dariusz has reported that the incidence of POCD in orthopedic subjects varies from $16 \%$ to $45 \%$ (16). The incidence of POCD in our study was $19.2 \%$ (55/287), which was quite similar to $19.6 \%$ reported by Rong et al.(14). As verified repeatedly, increased age is significantly associated with the increased risk of developing POCD (17), which is in accordance with our results of univariate analysis. Preexisting cognitive impairment has been reported to be closely associated with POCD in subjects undergoing hip joint replacement (18). Our univariate analysis also indicated preoperative MMSE score as a risk factor for POCD development. The close correlation between inflammatory marker concentrations, especially CRP, and POCD has been widely revealed $(19,20)$, which supports the pathogenic role of inflammation in POCD. However, our results from the final multivariate logistic regression analysis didn't support the predictive roles of these three parameters in POCD. In our opinion, the differences in sample size, inclusion and exclusion criteria, patient characteristics, preoperative comorbidities and the time of blood sampling might be possible explanations for the different conclusions.

In this present study, elevated serum pNF-H positivity was significantly associated with an increased risk of POCD in elderly subjects undergoing hip joint replacement. This finding suggested a potential application of serum pNF-H as a predictor for POCD development. As proved by previous studies, pNF-H may serve as an effective and reliable biomarker for CNS damage $(6,9)$. Our results directly revealed that POCD might be associated with anatomical damage in CNS. Previous studies have revealed the close association between pNF-H expression and the severity of spinal cord injury (5). Serum pNF-H has also been suggested as an effective biomarker for therapeutic efficacy evaluation among patients with spinal cord injury (21). Elevated pNF-H expressions are widely observed in those patients with supraspinal CNS damage caused by various conditions, such as acute intracerebral hemorrhage (22), hypoxic-ischemic encephalopathy (23) and febrile seizures (24).

Increased serum pNF-H concentrations are also observed in patients undergoing chemotherapy for breast cancer, suggesting the application of pNF-H as a potential biomarker of neural damage induced by chemotherapy (9). A close correlation between biomarkers of brain damage and POCD has been reported 
in orthopedic patients by previous literature (16), which strongly suggests the critical role of brain damage in POCD development. A recent report has revealed that PNF-H levels in patients undergoing operations for cervical compressive myelopathy are not elevated (25). In respect to our results, serum pNF-H may serve as a good candidate for POCD assessment. It might be beneficial to investigate the involved mechanisms using pNF-H as a surrogate biomarker for POCD development. The use of pNF-H detection as the potential biomarker for POCD can help to elucidate the potential role of anatomical brain damage in the mechanisms of POCD.

\section{Conclusisons}

In conclusion, our results revealed an increased preoperative serum pNF-H expression was an independent risk factor for POCD development in elderly subjects undergoing hip joint replacement, suggesting the close association between anatomical damage in CNS and POCD.

\section{Abbreviations}

POCD, postoperative cognitive dysfunction; ISPOCD, International Study of Postoperative Cognitive Dysfunction; pNF-H, phosphorylated neurofilament heavy subunit-H; CNS, central nervous system; ASA, American Society of Anesthesiologists; MMSE, Mini-Mental State Examination; BMI, Body Mass Index; ACE, angiotensin-converting enzyme; CRP, C-reactive protein; IL-6, interleukin-6; TNF-a, tumor necrosis factor-a; ELISA, enzyme-linked immuno sorbent assay; Cl: Confidence Interval; OR, Odds Ratio.

\section{Declarations}

This study was approved by the Medical Institutional Ethics Committee of Zhejiang province. The patients enrolled all presented written informed consent.

\section{Consent to Publish}

Yes.

\section{Competing interests}

None.

\section{Funding}

Health and Family Planning Commission of Zhejiang Province (2017KY137, 2017KY591, 2018KY693); Science and Technology Plan Project of Ningbo (2017C50060).

\section{Authors' contributions}


HF Z, JW Z participated in the conception and design, data collection, statistical analysis and wrote the manuscript. RC W, GR W and JP C participated in the conception and design and data collection.

\section{Availability of Data and Materials}

Yes.

\section{Acknowledgements}

None.

\section{References}

1. Bryson GL, Wyand A. Evidence-based clinical update: general anesthesia and the risk of delirium and postoperative cognitive dysfunction. Can J Anaesth. 2006;53(7):669-77.

2. Androsova G, Krause R, Winterer G, Schneider R. Biomarkers of postoperative delirium and cognitive dysfunction. Front Aging Neurosci. 2015;7:112.

3. Moller JT, Cluitmans P, Rasmussen LS, Houx P, Rasmussen H, Canet J, et al. Long-term postoperative cognitive dysfunction in the elderly ISPOCD1 study. ISPOCD investigators. International Study of Post-Operative Cognitive Dysfunction. Lancet. 1998;351(9106):857-61.

4. Lundstrom M, Edlund A, Bucht G, Karlsson S, Gustafson Y. Dementia after delirium in patients with femoral neck fractures. J Am Geriatr Soc. 2003;51(7):1002-6.

5. Hayakawa K, Okazaki R, Ishii K, Ueno T, Izawa N, Tanaka Y, et al. Phosphorylated neurofilament subunit NF-H as a biomarker for evaluating the severity of spinal cord injury patients, a pilot study. Spinal Cord. 2012;50(7):493-6.

6. Gresle MM, Liu Y, Dagley LF, Haartsen J, Pearson F, Purcell AW, et al. Serum phosphorylated neurofilament-heavy chain levels in multiple sclerosis patients. J Neurol Neurosurg Psychiatry. 2014;85(11):1209-13.

7. Lee S, Shea TB. The high molecular weight neurofilament subunit plays an essential role in axonal outgrowth and stabilization. Biol Open. 2014;3(10):974-81.

8. Galiano MR, Jha S, Ho TS, Zhang C, Ogawa Y, Chang KJ, et al. A distal axonal cytoskeleton forms an intra-axonal boundary that controls axon initial segment assembly. Cell. 2012;149(5):1125-39.

9. Natori A, Ogata T, Sumitani M, Kogure T, Yamauchi T, Yamauchi H. Potential role of pNF-H, a biomarker of axonal damage in the central nervous system, as a predictive marker of chemotherapyinduced cognitive impairment. Clin Cancer Res. 2015;21(6):1348-52.

10. Inoue R, Sumitani M, Ogata T, Chikuda H, Matsubara T, Kato S, et al. Direct evidence of central nervous system axonal damage in patients with postoperative delirium: A preliminary study of pNF-H as a promising serum biomarker. Neurosci Lett. 2017;653:39-44.

11. Murkin JM, Newman SP, Stump DA, Blumenthal JA. Statement of consensus on assessment of neurobehavioral outcomes after cardiac surgery. Ann Thorac Surg. 1995;59(5):1289-95. 
12. Silbert BS, Scott DA, Evered LA, Lewis MS, Kalpokas M, Maruff P, et al. A comparison of the effect of high- and low-dose fentanyl on the incidence of postoperative cognitive dysfunction after coronary artery bypass surgery in the elderly. Anesthesiology. 2006;104(6):1137-45.

13. Johnson T, Monk T, Rasmussen LS, Abildstrom H, Houx P, Korttila K, et al. Postoperative cognitive dysfunction in middle-aged patients. Anesthesiology. 2002;96(6):1351-7.

14. Lin R, Zhang F, Xue Q, Yu B. Accuracy of regional cerebral oxygen saturation in predicting postoperative cognitive dysfunction after total hip arthroplasty: regional cerebral oxygen saturation predicts POCD. J Arthroplasty. 2013;28(3):494-7.

15. Silverstein JH, Steinmetz J, Reichenberg A, Harvey PD, Rasmussen LS. Postoperative cognitive dysfunction in patients with preoperative cognitive impairment: which domains are most vulnerable? Anesthesiology. 2007;106(3):431-5.

16. Tomaszewski D. Biomarkers of Brain Damage and Postoperative Cognitive Disorders in Orthopedic Patients: An Update. Biomed Res Int. 2015;2015:402959.

17. Lewis $M$, Maruff $P$, Silbert $B$. Statistical and conceptual issues in defining post-operative cognitive dysfunction. Neurosci Biobehav Rev. 2004;28(4):433-40.

18. Silbert B, Evered L, Scott DA, McMahon S, Choong P, Ames D, et al. Preexisting cognitive impairment is associated with postoperative cognitive dysfunction after hip joint replacement surgery. Anesthesiology. 2015;122(6):1224-34.

19. Li X, Wen DX, Zhao YH, Hang YN, Mandell MS. Increase of beta-amyloid and C-reactive protein in liver transplant recipients with postoperative cognitive dysfunction. Hepatobiliary Pancreat Dis Int. 2013;12(4):370-6.

20. Liu X, Yu Y, Zhu S. Inflammatory markers in postoperative delirium (POD) and cognitive dysfunction (POCD): A meta-analysis of observational studies. PLoS One. 2018;13(4):e0195659.

21. Ueno T, Ohori Y, Ito J, Hoshikawa S, Yamamoto S, Nakamura K, et al. Hyperphosphorylated neurofilament NF-H as a biomarker of the efficacy of minocycline therapy for spinal cord injury. Spinal Cord. 2011;49(3):333-6.

22. Cai JY, Lu C, Chen MH, Ba HJ, Chen XD, Lin JH, et al. Predictive value of phosphorylated axonal neurofilament subunit $\mathrm{H}$ for clinical outcome in patients with acute intracerebral hemorrhage. Clin Chim Acta. 2013;424:182-6.

23. Douglas-Escobar M, Weiss MD. Biomarkers of hypoxic-ischemic encephalopathy in newborns. Front Neurol. 2012;3:144.

24. Matsushige T, Inoue H, Fukunaga S, Hasegawa S, Okuda M, Ichiyama T. Serum neurofilament concentrations in children with prolonged febrile seizures. J Neurol Sci. 2012;321(1-2):39-42.

25. Kato S, Chikuda H, Ohya J, Hayakawa K, Takeshita K, Tanaka S, et al. Phosphorylated neurofilament subunit levels in the serum of cervical compressive myelopathy patients. J Clin Neurosci. 2015;22(10):1638-42.

\section{Tables}


Table 1. Demographic and clinical data of patients with or without POCD

\begin{tabular}{|c|c|c|c|}
\hline Variables & $\mathrm{POCD} \square \mathrm{n}=55 \square$ & Non-POCD $\square n=232 \square$ & $P$-value \\
\hline Age (year) & $71.3 \pm 3.3$ & $70.2 \pm 2.9$ & 0.014 \\
\hline Gender, n (\%) & & & 0.393 \\
\hline Male & $20(36.4)$ & $99(42.7)$ & - \\
\hline Female & $35(63.6)$ & $133(57.3)$ & - \\
\hline BMI $\left(\mathrm{kg} / \mathrm{m}^{2}\right)$ & $23.1 \pm 2.7$ & $22.8 \pm 2.3$ & 0.401 \\
\hline ASA physical status, $\mathrm{n}(\%)$ & & & 0.431 \\
\hline II & $30(54.5)$ & $140(60.3)$ & - \\
\hline III & $25(45.5)$ & $92(39.7)$ & - \\
\hline Active smoker, n (\%) & $11(20.0)$ & $37(15.9)$ & 0.469 \\
\hline \multicolumn{4}{|l|}{ Preoperative comorbidities, n (\%) } \\
\hline Diabetes & $12(21.8)$ & $25(10.8)$ & 0.028 \\
\hline Hypercholesterolemia & $11(20.0)$ & $39(16.8)$ & 0.575 \\
\hline Hypertension & $16(29.1)$ & $40(17.2)$ & 0.046 \\
\hline Peripheral vascular disease & $4(7.3)$ & $14(6.0)$ & 0.734 \\
\hline History of myocardial infarct & $5(9.1)$ & $20(8.6)$ & 0.912 \\
\hline Preoperative MMSE (score) & $27.4 \pm 1.7$ & $28.0 \pm 1.9$ & 0.033 \\
\hline \multicolumn{4}{|l|}{ Preoperative medications } \\
\hline ACE inhibitors & $11(20.0)$ & $37(15.9)$ & 0.469 \\
\hline$\beta$-blockers & $8(14.5)$ & $34(14.7)$ & 0.984 \\
\hline Statins & $9(16.4)$ & $30(12.9)$ & 0.504 \\
\hline Duration of surgery (min) & $118.3 \pm 33.3$ & $107.8 \pm 29.1$ & 0.020 \\
\hline Duration of anesthesia (min) & $165.5 \pm 38.1$ & $153.8 \pm 32.5$ & 0.023 \\
\hline Recovery time (min) & $40.5 \pm 10.7$ & $40.2 \pm 11.1$ & 0.857 \\
\hline Estimated blood loss (ml) & $691.4 \pm 122.7$ & $654.2 \pm 105.7$ & 0.024 \\
\hline Blood transfusion, n (\%) & 13(23.6) & $34(14.7)$ & 0.106 \\
\hline
\end{tabular}

ASA, American Society of Anesthesiologists; BMI, Body Mass Index; MMSE, Mini-Mental State Examination; ACE, angiotensin-converting enzyme; POCD, Postoperative Cognitive Dysfunction. $P$-values were calculated by Chi-square test, Fisher exact test, Mann-Whitney U-test or t test. $* P$ value $<0.05$.

Table 2. The laboratory tests of patients with or without POCD 


\begin{tabular}{lccc}
\hline Variables & POCD $\llbracket \mathrm{n}=55 \square$ & Non-POCD $\llbracket \mathrm{n}=232 \square$ & P-value \\
\hline Hemoglobin $(\mathrm{g} / \mathrm{dL})$ & $11.4 \pm 1.7$ & $11.8 \pm 1.8$ & 0.136 \\
White blood cell $\left(\mathrm{x} 10^{9} / \mathrm{L}\right)$ & $7.3 \pm 2.0$ & $6.9 \pm 1.8$ & 0.148 \\
\hline CRP $(\mathrm{mg} / \mathrm{L})$ & $13.1 \pm 4.1$ & $12.0 \pm 3.4$ & 0.039 \\
\hline IL-6 $(\mathrm{pg} / \mathrm{mL})$ & $18.1 \pm 7.8$ & $17.5 \pm 6.7$ & 0.564 \\
\hline TNF- $\alpha(\mathrm{nmol} / \mathrm{L})$ & $8.0 \pm 2.2$ & $7.4 \pm 1.7$ & 0.028 \\
\hline Albumin $(\mathrm{g} / \mathrm{mL})$ & $40.1 \pm 4.2$ & $39.8 \pm 3.5$ & 0.583 \\
\hline Creatinine(mmol/L) & $84.1 \pm 18.1$ & $82.3 \pm 20.1$ & 0.544 \\
\hline Urea $(\mathrm{mmol} / \mathrm{L})$ & $6.7 \pm 2.1$ & $6.3 \pm 1.9$ & 0.170 \\
\hline pNF-H positivity, n (\%) & $10(18.2)$ & $16(6.9)$ & 0.009 \\
\hline
\end{tabular}

POCD, Postoperative Cognitive Dysfunction; CRP, C-reactive protein; IL-6, interleukin-6; TNF- $\alpha$, tumor necrosis factor- $\alpha$; pNF-H, phosphorylated neurofilament heavy subunit-H. $P$ values were calculated by Mann-Whitney U-test or t test. $* P$ value $<0.05$.

Table 3. Univariate and multiple logistic regression analyses for POCD

\begin{tabular}{lcccc}
\hline \multirow{2}{*}{ Variables } & \multicolumn{2}{c}{ Univariate } & Multivariate & \\
\cline { 2 - 5 } Age & OR(95\%CI) & P value & OR(95\%CI $)$ & $P$ value \\
\hline Diabetes & $2.12(1.05-4.41)$ & $0.028 *$ & $1.47(0.31-2.45)$ & 0.63 \\
\hline Hypertension & $1.22(0.58-2.51)$ & 0.54 & & \\
\hline Duration of surgery & $1.74(0.76-3.57)$ & 0.22 & & \\
\hline Duration of anesthesia & $1.95(0.87-4.35)$ & 0.11 & & \\
\hline Preoperative MMSE & $0.41(0.19-0.92)$ & $0.022 *$ & $0.66(0.31-1.52)$ & 0.38 \\
\hline Estimated blood loss & $1.12(0.83-1.49)$ & 0.16 & & \\
\hline CRP & $2.67(1.41-3.94)$ & $0.025 *$ & $1.41(0.75-2.84)$ & 0.17 \\
\hline TNF- $\alpha$ & $1.05(0.73-1.44)$ & 0.85 & & \\
\hline pNF-H positivity & $2.49(1.69-3.74)$ & 0.009 & $2.03(1.21-3.29)$ & 0.012 \\
\hline
\end{tabular}

POCD, Postoperative Cognitive Dysfunction; CRP, C-reactive protein; TNF- $\alpha$, tumor necrosis factor- $\alpha$; MMSE, Mini-Mental State Examination; CI: Confidence Interval; OR, Odds Ratio. $* P$ value $<0.05$. 